\title{
Deficiencia de 3-hidroxiacil coA deshidrogenasa de cadena larga, asociación con HELLP y hallazgos en la espectroscopia por resonancia magnética \\ Long chain 3-hydroxyacyl-coA dehydrogenase deficiency, asociation with HELLP and magnetic resonance spectroscopy findings
}

\author{
Dra. Noelia Deltetto ${ }^{a}$ Dra. Clarisa Maxit ${ }^{a}$ Dra. Delfina Marchione ${ }^{b}$, Dra. Marina Szlagoc, \\ Bioq. Andrea Schenone ${ }^{c}$, Dra. Cristina H. Besada ${ }^{d}$, Dra. María Vaccarezza y Dr. Guillermo Agosta ${ }^{a}$
}

\begin{abstract}
RESUMEN
La deficiencia de 3-hidroxiacil coA deshidrogenasa de cadena larga (LCHAD) es uno de los trastornos de la betaoxidación de ácidos grasos. La presentación clínica más frecuente incluye trastornos de conciencia, hipoglucemia y disfunción hepática gatillados por ayuno prolongado o infecciones. Una vez desencadenada, la crisis metabólica presenta alta mortalidad. El síndrome HELLP y la hepatitis grasa aguda del embarazo (AFLP) son trastornos del tercer trimestre del embarazo. Se ha asociado estas enfermedades durante la gestación con defectos hereditarios de la betaoxidación en el feto. Comunicamos el caso clínico de un trastorno de beta oxidación (deficiencia de LCHAD) asociado a HELLP materno. Describimos como hallazgos en la resonancia magnética espectroscópica un pico de ácido láctico y lípidos significativo. La pesquisa de estos trastornos de la betaoxidación al nacimiento, ante el antecedente de HELLP materno, permite el diagnóstico de la enfermedad previo al desarrollo de los síntomas.

Palabras clave: deficiencia de LCHAD, síndrome HELLP, pesquisa neonatal, resonancia magnética espectroscópica.
\end{abstract}

\section{SUMMARY}

LCHAD deficiency is a disorder of fatty acid beta oxidation. The most common clinical presentation includes disorders of consciousness, hypoglycemia and liver dysfunction triggered by prolonged fasting or infection. Once a metabolic crisis is triggered, there is a high mortality. HELLP syndrome and acute fatty liver failure of pregnancy (AFLP) are disorders of the third trimester of pregnancy. These diseases have been associated during pregnancy with hereditary defects of beta-oxidation in the fetus. We report a case of beta-oxidation disorder (LCHAD deficiency) associated with maternal HELLP. We described a peak of lipid and lactic on magnetic resonance spectroscopic

a. Servicio de Neurología Infantil. Hospital Italiano de Buenos Aires.

b. Sección Metabolismo. Servicio de Clínica Pediátrica. Hospital Italiano de Buenos Aires.

c. Laboratorio FESEN. Errores Congénitos del Metabolismo. Buenos Aires.

d. Servicio de Diagnóstico por Imágenes. Hospital Italiano de Buenos Aires.

Correspondencia:

Dra. Noelia Deltetto: noelia.deltetto@hospitalitaliano.org.ar ndeltetto@hotmail.com

Conflicto de intereses: Ninguno que declarar.

Recibido: 21-12-2011

Aceptado: 22-2-2012 of this patient. The investigation of these beta-oxidation disorders at birth, with a history of maternal HELLP, allows the diagnosis of the disease prior to developing symptoms.

Key words: LCHAD deficiency, HELLP syndrome, neonatal screening, magnetic resonance spectroscopic.

http:/ /dx.doi.org/10.5546/aap.2012.e63

Los trastornos de la betaoxidación de ácidos grasos constituyen un grupo heterogéneo de enfermedades con una frecuencia de 1 en 8000 nacidos vivos. ${ }^{1}$ La deficiencia de la enzima 3-hidroxiacil coA deshidrogenasa de cadena larga (LCHAD) es uno de estos trastornos.

El síndrome HELLP consiste en hemólisis, elevación de enzimas hepáticas y plaquetopenia; su prevalencia estimada en los EE.UU. de 5 en 1000 embarazos. La hepatitis grasa aguda del embarazo (AFLP) ocurre con una prevalencia estimada de 1 en 13000 gestaciones. ${ }^{3}$ Consiste en elevación de enzimas hepáticas, prolongación del tiempo de protrombina, hipoglucemia, hiperamoniemia, aumento de urea, creatinina, leucocitos, y posibilidad de desencadenamiento de una coagulación intravascular diseminada. Ambos trastornos presentan mecanismos fisiopatológicos comunes.

Varios trabajos en la bibliografía asocian estos trastornos maternos (AFLP o HELLP), cuya etiología es desconocida, con defectos hereditarios de la betaoxidación, entre ellos la deficiencia de LCHAD. ${ }^{1-7}$

Comunicamos el caso de un paciente con deficiencia de LCHAD y sus hallazgos en la resonancia magnética con espectroscopia de cerebro (ERM).

\section{Informe del caso}

Lactante varón de 5 meses de edad cronológica, nacido de 31 semanas por cesárea de urgencia por síndrome HELLP materno. Permaneció en neonatología durante 20 días para nutrición. El crecimiento pondoestatural permaneció en percentilos 25-50 y la maduración acorde a edad corregida. 
A las $48 \mathrm{~h}$ previas a su ingreso presentó rechazo alimentario e hipotonía, por lo que consultaron al hospital regional, donde se realizaron cultivos de sangre, orina y líquido cefalorraquídeo. Se inició tratamiento con ceftriaxona a $100 \mathrm{mg} / \mathrm{kg} /$ día.

Por desmejoría clínica, se deriva a un centro de mayor complejidad.

Ingresa a UCIP en paro respiratorio, bradicardia e hipotermia, Glasgow 3/15. Las maniobras de reanimación avanzada logran restablecer su función cardiorrespiratoria.

El laboratorio de ingreso evidenciaba: hipoglucemia con cetonas negativas en orina, acidosis metabólica, ácido láctico elevado y elevación de enzimas hepáticas (Tabla 1).

Tomografía axial computada de cerebro: normal.

A nivel respiratorio presentó trastorno grave de la oxigenación, con requerimiento de ARM. Hemodinamicamente permaneció inestable con requerimiento de inotrópicos. Presentó derrame pericárdico e hipertrofia grave de ventrículo izquierdo. Se constató insuficiencia hepática, con valores de enzimas y síntesis relativamente estables. Se indicó antibioticoterapia de amplio espectro. Los cultivos resultaron negativos. Se aportó alto flujo de glucosa con goteo de insulina para intentar controlar la crisis metabólica. Dada una mejoría clínica inicial, se solicitó resonancia magnética (RM) de cerebro con espectroscopia. La RM no evidenció anormalidad en el parénquima cerebral. Se menciona como hallazgo asociado en la ERM cerebral un pico de lactato y lípidos significativo (Figuras 1 y 2).

TABLA 1. Laboratorio al ingreso a nuestro hospital

\begin{tabular}{ll}
\hline Laboratorio & Valores \\
\hline Glucemia & $<20 \mathrm{mg} / \mathrm{dl}$ \\
Hematócrito & $21,5 \%$ \\
Leucocitos & $5940 / \mathrm{mm}^{3}$ \\
Plaquetas & $17100 / \mathrm{mm}^{3}$ \\
Estado ácido básico & $\mathrm{pH} 7,31 / / \mathrm{pCO}_{2} 36 \mathrm{mmHg}$ \\
& $\mathrm{EB}-7 \mathrm{mmol} / \mathrm{L} / / \mathrm{Bic} 18,5 \mathrm{mmol} / \mathrm{L}$ \\
Hepatograma & $\mathrm{BT} 3,8 \mathrm{mg} / \mathrm{dl} / / \mathrm{BD} 2,6 \mathrm{mg} / \mathrm{dl}$ \\
& $\mathrm{GOT} 86 \mathrm{UI} / \mathrm{L} / / \mathrm{GPT} 63 \mathrm{UI} / \mathrm{L}$ \\
Amoniemia & $143 \mathrm{mcg} / \mathrm{dl}(\mathrm{VN} 19-82)$ \\
Coagulación & $\mathrm{TP} \mathrm{y} \mathrm{KPTT} \mathrm{incoagulable}$ \\
Ionograma & $\mathrm{Na} 132 / \mathrm{K} \mathrm{3,8} / \mathrm{Cl} \mathrm{109}$ \\
Función renal & Uremia 29 mg/dl \\
Ácido láctico arterial & Creatininemia 0,36 mg/d $\mathrm{mmol} / \mathrm{L}$ (VN 0,5-2,5) \\
\hline
\end{tabular}

Con el cuadro clínico y el antecedente materno de síndrome HELLP se solicitó estudio metabólico. Se realizó diagnóstico de trastorno congénito de la betaoxidación de los ácidos grasos, con los siguientes hallazgos:

Ácidos orgánicos en orina: marcado aumento de 3 hidroxiácidos dicarboxílicos.

Acilcarnitinas en sangre: marcado aumento de acilcarnitinas y 3 hidroxiacilcarnitinas de cadena larga.

Estos resultados son compatibles con deficiencia de LCHAD y / o deficiencia de proteína trifuncional mitocondrial (PTM).

Se logró hacer el estudio retrospectivamente en el cartón de pesquisa neonatal, con los mismos resultados.

Se confirmó el diagnóstico de deficiencia de LCHAD con biología molecular para detección de la mutación genética. La mutación hallada está en el gen HADHA localizado en el cromosoma 2 (2p23.3) (OMIM *600890). Este gen codifica para la subunidad alfa de la PTM, donde tiene lugar la acción enzimática de LCHAD. La mutación encontrada fue 1528G >C (E510Q) en la subunidad alfa de la PTM, que ocasiona un cambio de aminoácido por otro, generando pérdida de la actividad enzimática.

A pesar del tratamiento de su crisis metabólica, el niño desarrolló disfunción orgánica múltiple y al $6^{\circ}$ día de internación falleció.

\section{DISCUSIÓN}

Dos aspectos nos parecen interesantes en este informe. El primero es el antecedente de HELLP materno en un niño con deficiencia de LCHAD, asociación ya descripta en la bibliografía aunque

Figura 1. Resonancia magnética de cerebro

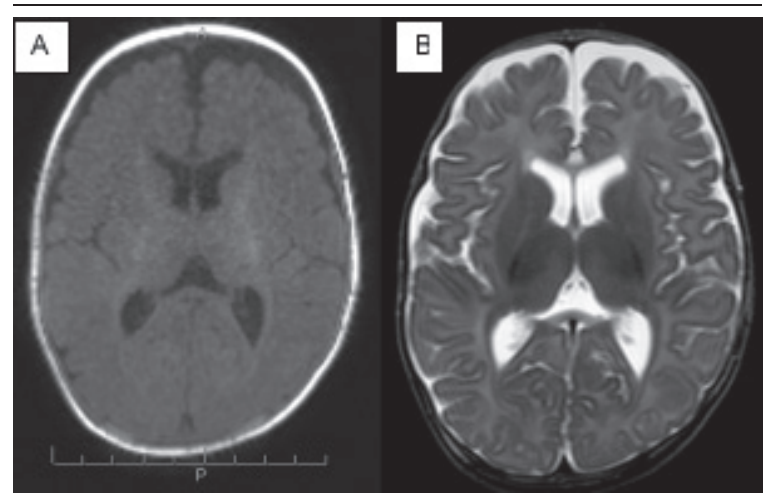

Resonancia magnética de cerebro: imágenes normales. A. Plano axial ponderadoen T1. B. Plano axial ponderado en T2. 
controvertida ${ }^{1,-5,8-10}$. El segundo aspecto está dado por los hallazgos observados en ERM de cerebro.

Las formas de presentación clínica de la deficiencia de LCHAD son variables:

a) Síndrome de tipo "Reye like" con hipoglucemia, hígado graso y coma,

b) Muerte súbita,

c) Miocardiopatía hipertrófica o dilatada,

d) Miopatía.

El diagnóstico presuntivo se basa en el cuadro clínico, asociado a un perfil característico de acilcarnitinas en sangre y de ácidos orgánicos en orina.

La confirmación diagnóstica es posible al medir la actividad enzimática de LCHAD, o bien por el análisis de mutación genética.

En 1995, Strauss comunicó las bases moleculares de niños con deficiencia de LCHAD asociados a madres con AFLP. ${ }^{4}$

FIGURA 2. Resonancia magnética espectroscópica.
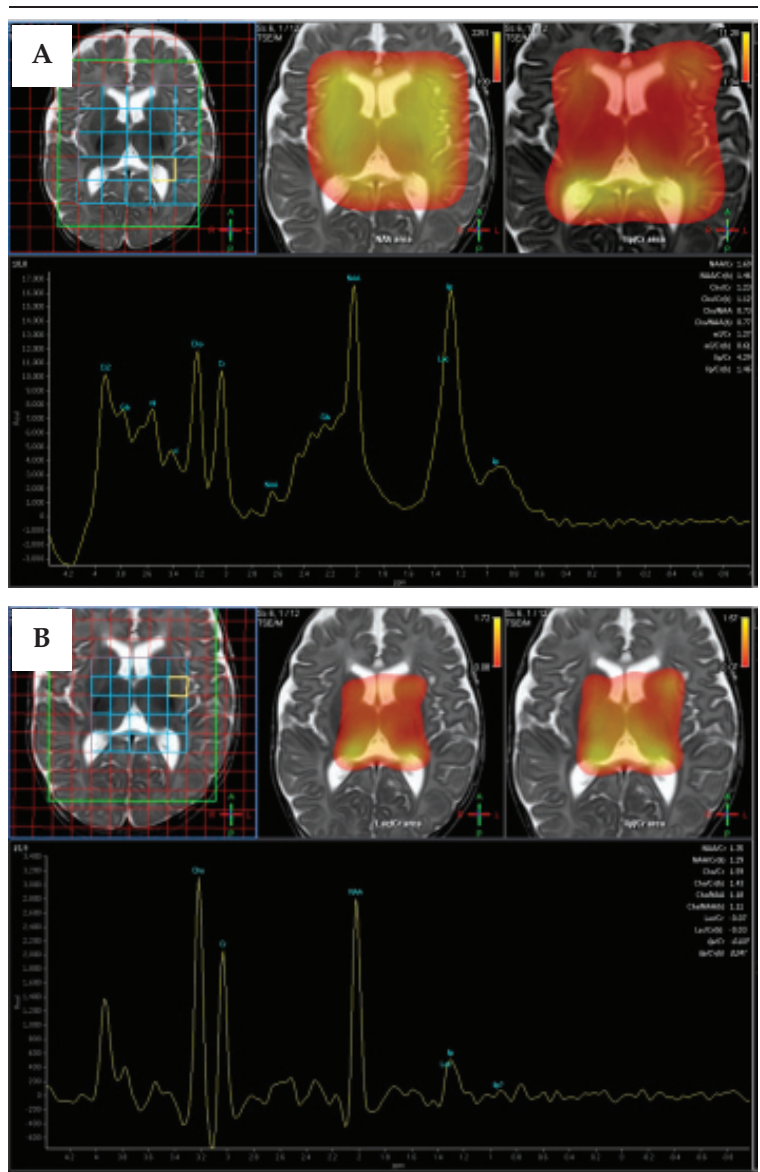

Espectroscopia multivoxel de cerebro. A. Con tiempo de eco corto (30) y B. Intermedio (135). Se observa un pico de láctico y lípidos significativos.
En 1999, Ibdah halló que el 79\% de madres con niños afectados por trastornos de la betaoxidación habían presentado HELLP. ${ }^{5}$

En 2002, lbdah publicó una asociación significativa entre AFLP y mutación E474Q en el feto. ${ }^{1}$

En 2006, Shih concluyó que la enfermedad hepática materna es significativamente más alta en los embarazos con fetos afectados por defectos de la betaoxidación que en controles de la población general. ${ }^{6}$

Sin embargo, en la bibliografía existen trabajos que no hallaron asociación entre el desarrollo de HELLP y trastornos de la betaoxidación. Mansouri y cols., no encontraron la mutación en mujeres con diagnóstico de AFLP. ${ }^{11}$ Den Boer y cols., estudiaron mujeres con antecedentes de HELLP y solo una fue heterocigota para la mutación. ${ }^{10} \mathrm{Ho}-$ lub y cols., no detectaron alteración en el perfil de acilcarnitinas, ni la mutación más común para LCHAD en niños nacidos de madres con HELLP. ${ }^{7}$ Mutze y cols., publicaron la ausencia de la mutación fetal y materna en embarazos complicados con HELLP. 8

La mortalidad comunicada de los niños con deficiencia completa de PTM o LCHAD ha sido del $75-90 \% .{ }^{5}$ El tratamiento dietético temprano en estos niños reduce drásticamente la mortalidad. La implementación de la pesquisa neonatal ha reducido en forma significativa la morbilidad y la mortalidad de los trastornos de la betaoxidación. ${ }^{9}$

La ERM puede ser de utilidad en el diagnóstico de pacientes con sospecha de errores congénitos del metabolismo. En la ERM existen picos de sustancias normales en el parénquima cerebral, que resuenan en determinadas partes por millón (ppm) del eje $x$ del patrón espectroscópico (entre ellos se destacan un pico de colina, creatina y $\mathrm{N}$-acetil aspartato). Frente a la cantidad de defectos metabólicos, son pocos los patrones de metabolitos específicos anormales identificados en ERM, como por ejemplo en la enfermedad de Canavan, donde existe un aumento del pico de $\mathrm{N}$-acetil aspartato, o en la deficiencia de creatina cerebral, donde hay una disminución del pico de creatina. ${ }^{12} \mathrm{El}$ pico de láctico y de lípidos resuenan a 1,33 ppm en la ERM y son siempre anormales. Para distinguir uno de otro es menester realizar imágenes con los 2 tiempos de eco (intermedio $135 \mathrm{~ms}$ o largo $270 \mathrm{~ms}$ y corto $30 \mathrm{~ms}$ ), ya que el pico de láctico posee una morfología de doblete y es negativo en tiempo de eco intermedio o largo, y se positiviza en tiempo de eco corto, mientras que el pico de lípidos es positivo en ambos tiempos. 
El hallazgo de un pico de lípidos en la ERM en este paciente podría ser explicado por el acúmulo de metabolitos grasos anormales en el parénquima cerebral, dada la falla enzimática en el ciclo oxidativo de los ácidos grasos de cadena larga. ${ }^{13-15}$. El pico de láctico se observa en otras entidades metabólicas, como trastornos mitrocondriales, y en aquellas por depósitos de sustancias patológicas por disfunción mitocondrial. ${ }^{12}$ Estos hallazgos aportan algo más al conocimiento y a las posibilidades diagnósticas en este grupo de patologías; cabe a los estudios futuros demostrar la incidencia del cuadro y la utilidad de este enfoque.

Querríamos enfatizar que, en nuestra opinión, sería recomendable que los recién nacidos, hijos de madres que presentaron AFLP o HELLP, puedan acceder a la pesquisa de defectos de la betaoxidación. Este punto es crítico para el diagnóstico temprano y el tratamiento adecuado, ya que podría evitarse la crisis metabólica que en estos lactantes conlleva alta mortalidad.

\section{BIBLIOGRAFÍA}

1. Ibdah JA, Strauss AW, Zhao Y, Yamada J, Yang Z. Prospective screening for pediatric mitochondrial trifunctional protein defects in pregnancies complicated by liver disease. JAMA 2002;288(17): 2163-6.

2. den Boer M, Wanders R, Morris A, Ijist L, et al. Long chain 3 hydroxyacyl coA dehydrogenase deficiency: clinical presentation and follow up of 50 patients. Pediatrics 2002;109(1):99-104.

3. Ibdah, JA. Acute fatty liver of pregnancy: an update on pathogenesis and clinical implications. World J Gastroenterol 2006;12(46):7397-404.

4. Strauss AW, Shapiro S, Gibson B, Bennett MJ, et al. The molecular basis of pediatric long chain 3-hydroxyacylCoA dehydrogenase deficiency associated with maternal acute fatty liver of pregnancy. Proc Natl Acad SCI USA 1995;92(3):841-5.
5. Idbah JA, Bennett MJ, Rinaldo P, Zhao Y, Gibson B, Sims HF, Strauss AW. A fetal fatty-acid oxidation disorder as a cause of liver disease in pregnant women. $N$ Engl J Med 1999;340(22):1723-31.

6. Shih VE, Larson C, Wilkins-Haug LE, Levy HL, Browning MF. Fetal fatty acid oxidation defects and maternal liver disease in pregnancy. Obstet Gynecol 2006;107(1):115-20.

7. Holub M,Stockler-Ipsiroglu S, PollakA, Muhl A, et al. Lack of correlation between fatty acid oxidation disorders and haemolysis, elevated liver enzymes, low platelets (HELLP) syndrome? Acta Paediatr 2005;94(1):48-52.

8. Mütze S, Ahillen I, Rudnik-Schoeneborn S, Eggermann T, et al. Neither maternal nor fetal mutation (E474Q) in the alpha-subunit of the trifunctional protein is frequent in pregnancies complicated by HELLP syndrome. J Perinat Med 2007;35(1):76-8.

9. Spiekerkoetter U. Mitochondrial fatty acid oxidation disorders: clinical presentation of long-chain fatty acid oxidation defects before and after newborn screening. J Inherit Metab Dis 2010;33(5):527-32.

10. Den Boer ME, Ijlst L, Wijburg F, Oostheim W, et al. Heterozygosity for the common LCHAD, mutation (1528G $>$ C) is not a major cause of HELLP syndrome and the prevalence of the mutation in the Dutch population is low. Pediatr Res 2000;48(2):151-4

11. Mansouri A, Fromenty B, Durand F, Degott C, et al. Assessment of the prevalence of genetic metabolic defects in acute fatty liver of pregnancy. J Hepatol 1996;25(5):781-2.

12. Cecil KM. MR Spectroscopy of metabolic disorders. Neuroimaging Clin N Am 2006;16(1):87-116.viii.

13. Roomets E, Lundbom N, Pihko H, Heikkinen S, Tyni T. Lipids detected by brain MRS during coma caused by carnitine palmitoyltransferase 1 deficiency. Neurology 2000;67(8):1516-7.

14. Pirgon O, Aydin K, Atabek ME. Proton magnetic resonance spectroscopy findings and clinical effects of montelukast sodium in a case with Sjögren-Larsson syndrome. J Child Neurol 2006;21(12):1092-5.

15. Groenendaal F, Bianchi MC, Battini R, Tosetti M, et al. Proton magnetic resonance spectroscopy (1H-MRS) of the cerebrum in two young infants wirh Zellweger syndrome. Neuropediatrics 2001;32(1):23-7. 